\title{
Effects of Arbitrarily Spaced Subcarriers on Detection Performance in OFDM Radar
}

\author{
Johannes Fink, Martin Braun and Friedrich K. Jondral \\ Communications Engineering Lab, Karlsruhe Institute of Technology (KIT), Karlsruhe, Germany \\ E-mail: \{johannes.fink, martin.braun, friedrich.jondral\}@kit.edu
}

\begin{abstract}
Orthogonal frequency division multiplexing (OFDM) is a transmission technique which divides a given frequency band into subbands such that the subcarriers are mutually orthogonal. Because of its favourable transmission properties, OFDM is widely used in wireless communications. Recently it was shown that it is possible to extract information about distance and speed of obstacles from OFDM scatterings as in a radar system. The combination of communications and radar in a single OFDM system for automotive applications was named OFDM Radar. In this paper a simple multi user access scheme for OFDM Radar is proposed and its implications on radar detector design are discussed. The proposed scheme is a variant of OFDMA based on the idea that each user chooses independently a subset of the available OFDM carriers for transmission. The chosen subcarriers can be spaced arbitrarily. A suitable detector which is furthermore capable of multiple target detection is derived and its performance in various scenarios is demonstrated using Monte Carlo simulations.
\end{abstract}

Index Terms-OFDM Radar, OFDMA, detection, multi user access, multiple target detection, mobile ad-hoc network

\section{INTRODUCTION}

Recently, a novel concept for automotive radar, which is based on the idea of utilizing the same OFDM signal for both communications and radar, was proposed in [1]. As shown in [2], the radar signal processing can be accomplished by exploiting the two-dimensional structure of the OFDM frames by means of (I)FFTs, if either all subcarriers or a subset of equidistant spaced subcarriers are used.

In an automotive application, multi user access (MUA) is a critical component. As the proposed network is of mobile ad-hoc type, a suitable and robust MUA strategy is needed. In this paper we propose a strategy of stochastic nature that does neiter rely on spectrum sensing techniques nor information exchange between the users. The approach examined is to separate the users in frequency domain via the OFDM subcarriers as in OFDMA. However, instead of using sets of equidistantly spaced subcarriers, the idea is that each user chooses a random set of subcarriers with the same probability for each subcarrier to be chosen. Consequently their spacing will be arbitrary, which drastically reduces the chance of two users choosing exactly the same set. Thus, if the signal-processing can be adapted to be able to deal with these constraints, the chance for system outage will diminish.

The question addressed in this paper is how the radar signal processing has to be performed when subcarriers are no longer equidistant and how partial interference affects the detector performance.

\section{SignAL MODEL}

As shown in [2], the OFDM signal structure allows to describe radar echos as two orthogonal sinusoids modulating the originally transmitted signal. Thus, the detection problem can be transferred to the problem of detecting sinusoids in noisy signal samples, which is a topic in spectral analysis. This connection will be shown briefly hereafter.

\section{A. Radar target modeling}

In the context of OFDM Radar, the quantities of interest are range $R$ and relative velocity $v_{\text {rel }}$ of a target. These quantities determine the delay

$$
\tau=\frac{2 R}{c_{0}}
$$

and the Doppler shift

$$
f_{D}=f_{c} \frac{2 v_{\mathrm{rel}}}{c_{0}}
$$

of the received radar echo. The constant $c_{0}$ is the speed of light and $f_{c}$ is the center frequency of the signal. Eq. (2) is actually an approximation, which holds for signals with relative bandwidth $\frac{B}{f_{c}} \ll 1$. For details on parametrization of OFDM Radar systems, see [3]. The third influence of radar targets on the signal is attenuation by a factor $b \in \mathbb{C}$.

Several targets contribute to a linear superposition of delayed, frequency shifted and attenuated versions of the originally transmitted signal $s(t)$ at the receiver. Assuming a total number of $H$ targets and additive white gaussian noise (AWGN) with the realization $n(t)$, the received signal can be described as

$$
y(t)=\sum_{h=0}^{H-1} b_{h} s\left(t-\tau_{h}\right) \mathrm{e}^{j 2 \pi f_{D, h} t}+n(t) .
$$

\section{B. OFDM signal}

For the scope of this paper, a whole OFDM frame consisting of $L$ OFDM symbols will be considered [4]. The total number of available subcarriers shall be $N_{T}$ out of which each user is allowed to draw a number of $K$ subcarriers for transmission. Furthermore we consider rectangularly shaped orthogonal pulses

$$
\Psi_{c_{k}, l}(t)=\frac{1}{\sqrt{T_{O}}} \mathrm{e}^{j 2 \pi f_{c_{k}} t} \operatorname{rect}\left(\frac{t-l T_{O}}{T_{O}}\right)
$$

with a OFDM symbol duration of $T_{O}$ and $l=0,1, \ldots, L-1$ indicating the OFDM symbol number within the OFDM frame. 
In (4), $f_{c_{k}}=f_{0}+c_{k} \Delta f$ is the center frequency of the $c_{k}$-th subcarrier with $c_{k} \in 0,1, \ldots N_{T}-1$ and $k=0,1, \ldots K-1$ indicating the subcarrier number within the chosen subset. The center frequency of the first subcarrier shall be $f_{0}$ and the subcarrier spacing $\Delta f$. Furthermore, the rectangle function shall be defined as

$$
\operatorname{rect}\left(\frac{t}{T}\right)= \begin{cases}1, & 0 \leq t<T \\ 0, & \text { otherwise }\end{cases}
$$

Neglecting unused subcarriers and denoting the modulation symbols as $a_{c_{k}, l} \in \mathcal{A} \subset \mathbb{C}$, where $\mathcal{A}$ is the modulation alphabet, the transmitted signal can be written as

$$
s(t)=\sum_{l=0}^{L-1} \sum_{k=0}^{K-1} a_{c_{k}, l} \Psi_{c_{k}, l}(t), \quad 0 \leq t \leq L T_{O} .
$$

Inserting (6) into (3) and expressing $b_{h}=\beta_{h} \mathrm{e}^{j \phi_{h}}$ yields

$$
\begin{array}{r}
y(t)=\sum_{h=0}^{H-1} \sum_{l=0}^{L-1} \sum_{k=0}^{K-1} \beta_{h} \mathrm{e}^{j \phi_{h}} a_{c_{k}, l} \frac{1}{\sqrt{T_{O}}} \mathrm{e}^{j 2 \pi f_{c_{k}}\left(t-\tau_{h}\right)} \\
\operatorname{rect}\left(\frac{t-\tau_{h}-l T_{O}}{T_{O}}\right) \mathrm{e}^{j 2 \pi f_{D, h} t}+n(t) .
\end{array}
$$

Further details on the signal model for OFDM radar can be found in [5] and [6].

By sampling and applying the inverse OFDM operation, the continuous model of (7) is turned into a discrete one where the samples obtained can be arranged to form a discrete matrix $\mathbf{F}_{\mathrm{Rx}} \in \mathbb{C}^{K \times L}$ with the elements

$$
\left(\mathbf{F}_{\mathrm{Rx}}\right)_{k, l}=\sum_{h=0}^{H-1} \beta_{h} \mathrm{e}^{j \phi_{h}} a_{c_{k}, l} \mathrm{e}^{j 2 \pi l T_{O} f_{D, h}} \mathrm{e}^{-j 2 \pi c_{k} \tau_{h} \Delta f}+n_{k, l}
$$

and $n_{k, l}$ denoting the sampled noise realization. Dividing [8] by the known modulation symbols $a_{c_{k}, l}$ yields

$$
(\mathbf{F})_{k, l}=\sum_{h=0}^{H-1} \beta_{h} \mathrm{e}^{j \phi_{h}} \mathrm{e}^{j 2 \pi l T_{O} f_{D, h}} \mathrm{e}^{-j 2 \pi c_{k} \tau_{h} \Delta f}+w_{k, l},
$$

where $w_{k, l}=\frac{n_{k, l}}{a_{c_{k}, l}}$. Assuming a PSK modulation and uncorrelated symbols $a_{c_{k}, l}$, the noise statistic remains unaltered.

\section{DETECTOR}

Given the $K L$ noisy samples of the sum signal according to (9), the detector's task is to estimate the parameters $\beta_{h}, f_{D, h}$ and $\tau_{h} \forall h \in\{0,1, \ldots, H-1\}$. Estimation of phase $\phi_{h}$ is not treated in this paper.

In order to develop a method to solve the multiple detection problem, first the problem shall be solved for the case, when only one target is present, i.e., $H=1$. This can be done for example using a maximum likelihood (ML) approach as in [2]. Adaptions have to be made to take the arbitrary spacing of the subcarriers into account.

\section{A. Maximum likelihood detector for single sinusoid}

The starting point when making a ML approach is deploying the likelihood function, which for the given signal model is

$$
f(\mathbf{F} \mid \boldsymbol{\theta})=\prod_{k=0}^{K-1} \prod_{l=0}^{L-1} \frac{1}{\pi \sigma^{2}} \mathrm{e}^{-\frac{\left|(\mathbf{F})_{k, l}-\mathrm{e}^{j\left(2 \pi\left(l T_{O} f_{D}-c_{k} \tau \Delta f\right)+\phi\right)}\right|^{2}}{\sigma^{2}}},
$$

where $\boldsymbol{\theta}=\left(f_{D}, \tau, \phi\right)$ is the parameter vector.

Maximization of function (10) with regard to $\theta$ yields the estimate. This challenging task can be simplified at the cost of reduced accuracy by quantizing the frequency variables as in [2] according to

$$
f_{D, Q, m}=\frac{m}{M T_{O}}, \quad m=-\frac{M}{2},-\frac{M}{2}+1, \ldots, \frac{M}{2}-1
$$

and

$$
\tau_{Q, n}=\frac{n}{N \Delta f}, \quad n=0,1,2, \ldots, N-1 .
$$

In (11) and 12, the natural numbers $M \geq K$ and $N \geq L$ are tuning parameters which determine the accuracy of the frequency estimates on one hand and affect the computational burden on the other hand. The values $\xi_{1}=\frac{M}{K}$ and $\xi_{2}=\frac{N}{L}$ are called interpolation factors (IFs). The two IFs can differ from each other but in the simulations carried out for this paper they were always chosen so that $\xi_{1}=\xi_{2} \triangleq \xi$.

Inserting (11) and (12) into 10, taking the logarithm and rearranging yields the function

$$
\ell_{Q}\left(\mathbf{F} \mid \boldsymbol{\theta}_{Q}\right)=\Re\left\{\mathrm{e}^{j \phi}(\mathbf{B})_{m, n}\right\}
$$

with

$$
(\mathbf{B})_{m, n}=\sum_{k=0}^{K-1}\left(\sum_{l=0}^{L-1}(\mathbf{F})_{k, l} \mathrm{e}^{-j 2 \pi \frac{l m}{M}}\right) \mathrm{e}^{j 2 \pi \frac{c_{k} n}{N}},
$$

where $\boldsymbol{\theta}_{Q}=\left(f_{D, Q, m}, \tau_{Q, n}, \phi\right)$ is the parameter vector with quantized frequencies and $\Re\{\cdot\}$ denotes taking the real part of a complex number. If $\phi$ is unknown, then the discrete solution to the ML problem is the one that maximizes $\left|(\mathbf{B})_{m, n}\right|$ or alternatively $(\mathbf{C})_{m, n}=\left|\frac{1}{K L}(\mathbf{B})_{m, n}\right|^{2}$, where $\mathbf{C}$ resembles a two-dimensional periodogram.

In [2], it is pointed out, that $\mathbf{B}$ can be efficiently calculated by means of the (I)FFT: First, on each row of $\mathbf{F}$ a FFT has to be applied; then, on the resulting matrix, an IFFT of each column has to be calculated. Taking care of the nonequidistant samples (due to non-equidistant subcarriers) can be done either by expanding $\mathbf{F}$ with zero-rows for each possible sampling instant, where no sample is available and increasing the according IFFT size or evaluating the involved operations straight forward, i.e., without taking advantage of highly efficient (I)FFT algorithms, causing a higher computational load.

\section{B. Iterative detection of multiple sinusoids}

Although expression (14) was derived for the single target case, it may also be used for detection and parameter estimation, when there are multiple targets. As in one-dimensional periodograms, however, every peak in the periodogram induces 
a high amount of leakage. That is why a threshold detection directly applied to the periodogram will result in many false alarms. The leakage is due to the implicit windowing performed before calculating the (I)FFTs. Keeping in mind the effects of windowing [7], the following iterative threshold adaption strategy was developed to tackle this problem:

1) Calculate two-dimensional periodogram $\mathbf{C}$ of matrix $\mathbf{F}$.

2) Generate a threshold matrix $\mathbf{M}$ of the same size as $\mathbf{C}$ with each cell containing an individual threshold value for the associated periodogram cell. Initiate all cells with a common threshold value $\gamma$.

3) Of all periodogram values exceeding their associated threshold, find the maximum.

4) Put the according parameters as estimates in a results list.

5) Update the threshold matrix $M$ to incorporate the effects caused by leakage of the found peak.

6) Repeat steps 3 to 5 until $(\mathbf{M})_{i, j}>(\mathbf{C})_{i, j} \forall i, j$.

The initial threshold level $\gamma$ is set in order to achieve a given maximum false alarm rate, thus it is subject to the noise power $\sigma^{2}$ and can be calculated as in a Neyman-Pearson detector:

$$
P_{\mathrm{FA}}=\int_{\gamma} p_{\Upsilon}\left(\Upsilon \mid H_{0}\right) \mathrm{d} \Upsilon=1-F_{\Upsilon}\left(\gamma \mid H_{0}\right),
$$

where $H_{0}$ is the Null Hypothesis, $\Upsilon$ the detector statistic and $P_{\mathrm{FA}}$ the desired false-alarm rate. If the samples were chosen equidistantly, the values under hypothesis $H_{0}$ and complex, circular symmetric, zero-mean AWGN, would follow a $\chi^{2}$ distribution with two degrees of freedom:

$$
F_{\Upsilon}(y)= \begin{cases}1-\mathrm{e}^{-\frac{y}{2 \sigma^{2}}}, & y \geq 0 \\ 0, & y<0 .\end{cases}
$$

As explained in [8], in the case of non-equidistant samples, this statistic depends on the actually chosen sampling instants and cannot be generically derived analytically. For the simulations in this paper, the needed cumulative density functions were derived by means of Monte Carlo simulations.

The threshold update is done in an additive manner. To account for the spectral influence of detected sinusoids in the signal, a spectral mask $\mathbf{W}$ is calculated, appropriately scaled and then added to the threshold matrix $\mathbf{M}$ :

1) Initialization: Calculate $\gamma$ according to (15) and use to initialize threshold matrix: $\mathbf{M}^{1}=\gamma[\mathbf{1}]^{N \times M}$, where $[1]^{N \times M}$ denotes a $N \times M$ matrix of ones.

2) Search: $\left(n_{i}, m_{i}\right)=\max _{m, n}\left\{(\mathbf{C})_{n, m}>(\mathbf{M})_{n, m}^{i}\right\}$.

3) Threshold update: $(\mathbf{M})_{n, m}^{i+1}=(\mathbf{M})_{n, m}^{i}+\hat{P}_{i}(\mathbf{W})_{n, m}$, where $\hat{P}_{i}$ is the estimated power of target number $i$.

This procedure allows controlling the effects of sidelobes due to spectral leakage. However, there is a trade-off between false alarms and misses, tunable by the scaling factor $\hat{P}_{i}$.

As the signals to detect are sinusoids, their influence in the frequency domain is to relocate the spectral function of the window. Therefore, it is possible to calculate a prototype spectral window $\mathbf{W}_{\mathbf{P}} \in \mathbb{R}_{\geq 0}^{N \times M}$ once and then for each detected sinusoid scale and move it accordingly before adding to the threshold matrix. Because of the two orthogonal dimensions, the two-dimensional spectral mask $\mathbf{W}_{\mathbf{P}}$ can be calculated as dyadic product

$$
\mathbf{W}_{\mathbf{P}}=\mathbf{w}_{1} \mathbf{w}_{2}^{T}
$$

of two one-dimensional spectral masks $\mathbf{w}_{1}$ and $\mathbf{w}_{2}$. They can be calculated using the one-dimensional periodogram.

As the frequencies are discretized (see (11) and (12)), the straddle loss [7], i.e., the difference between the real power of the sinusoid from the estimated power, has to be taken into account when calculating the spectral mask (i.e., we have to be sure, that the adapted threshold is actually higher than the sidelobes of the regarded peak). Practically this can be done by first calculating $\mathbf{W}_{\mathbf{P}}$ using a very fine frequency grid and then resampling in a max-hold fasion.

Goal of designing $\mathbf{W}_{\mathbf{P}}$ is to eliminate the possibility of false alarms due to the convolved spectral window, while at the same time raising the threshold only as little as needed to not generate misses (present targets which are not detected). This leads to the following way of generating $\mathbf{w}_{1}$ and $\mathbf{w}_{2}$ :

1) Calculate a high resolution spectral window

$$
\tilde{W}(m) \triangleq \tilde{W}\left(\omega_{m}\right)=\frac{1}{K^{2}} \sum_{k=1}^{K}\left|\mathrm{e}^{-j \omega_{m} t_{k}}\right|^{2}
$$

where $m=0,1, \ldots, M-1$ whith $M \gg K$ and $\omega_{m} \in$ $[-\pi ; \pi)$.

2) Resample to the $N$-elements grid according to the rule

$$
\begin{array}{r}
W(n)=\max \left\{\tilde{W}\left(\frac{n M}{N}\right), \tilde{W}\left(\frac{n M}{N}+1\right), \ldots,\right. \\
\left.\tilde{W}\left(\frac{(n+1) M}{N}\right)-1\right\} .
\end{array}
$$

This procedure is visualized in fig. $1 \mathrm{a}$ and $1 \mathrm{~b}$

\section{RESULTS}

To evaluate the performance of the proposed detector, Monte Carlo simulations were performed. As the novelty of the detector is to deal with arbitrarily spaced subcarriers, it was compared with a detector proposed in [2] that requires equidistant subcarriers. As that detector originally was designed for single target detection, it was extended to the case of multiple targets in the same fashion as the detector described in this paper.

The results presented are detection statistics. In each Monte Carlo run, the number of false alarms and the number of detects per OFDM frame was determined. The values for detects and false alarms were averaged over a total of 1000 frames.

In each detection run, each target's parameters $v_{\text {rel }}$ and $R$ were drawn from a uniform distribution within the detectable ranges, whiche were in our scenario $v_{\text {rel }} \in$ $[-150 \mathrm{~m} / \mathrm{s} ;+150 \mathrm{~m} / \mathrm{s}]$ and $R \in[0 \mathrm{~m} ;+200 \mathrm{~m}]$. For the case of arbitrarily spaced subcarriers, the used subcarriers in each detection run were drawn from a uniform distribution across all subcarriers. The target false alarm rate used to calculate the 


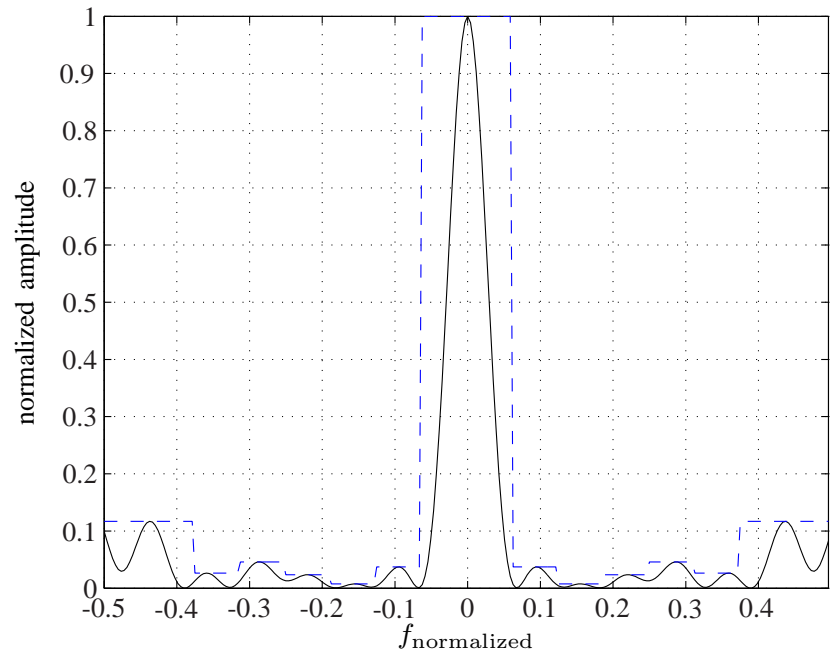

(a) Generation of threshold mask.

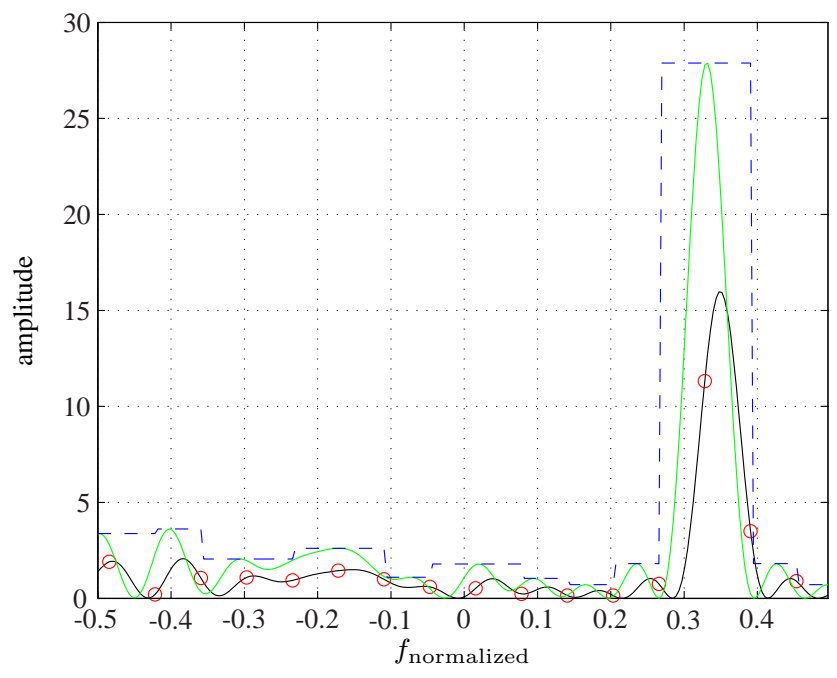

(b) Used threshold mask.

Fig. 1: Visualization of the resampling process to generate the threshold mask (blue) from 16 non-equidistant samples. The true window function is plotted in black. Red circles mark the calculated spectral components on the used DFT grid. The estimated spectral function is shown in green.

initial threshold was set to 0.1 per OFDM frame. If not stated otherwise, the number of targets was always set to 30. Further details on simulation parameters are summarized in table I]

\section{A. Influence of interpolation factor}

Fig. 2 shows the average number of detects and false alarms subject to the used subcarrier selection scheme (equidistant or random), as well as subject to the interpolation factor (IF).

One can see that the detection rate gets better as the IFs increase, as well as the differences between the two detectors diminish. The detector based on equidistant subcarriers performs slightly better but the detection rate of both detectors is above $90 \%$ for IFs greater than two.
TABLE I: Summary of simulation parameters

\begin{tabular}{ccc}
\hline parameter & symbol & Value \\
\hline subcarrier modulation scheme & - & BPSK \\
total number of OFDM subcarriers & $N_{T}$ & 1024 \\
number of OFDM symbols per Frame & $L$ & 256 \\
center frequency & $f_{c}$ & $24 \mathrm{GHz}$ \\
subcarrier spacing & $\Delta f$ & $90.9 \mathrm{kHz}$ \\
guard interval fraction & - & $\frac{1}{8}$ \\
EIRP & - & $20 \mathrm{dBm}$ \\
number of subcarriers used & $K$ & 256 \\
noise figure of receiver & $F$ & $10 \mathrm{~dB}$ \\
crosstalk attenuation & - & $-50 \mathrm{~dB}$ \\
\hline
\end{tabular}

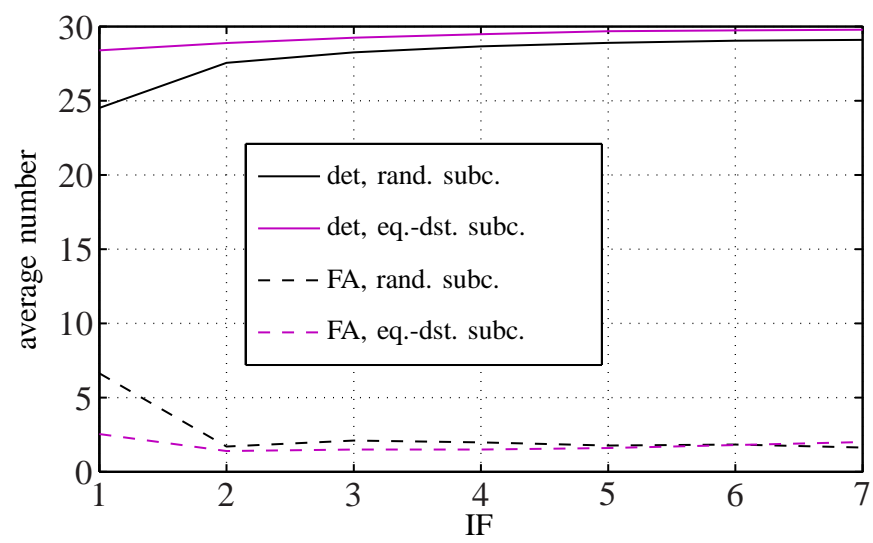

Fig. 2: Detects (det) and false-alarms (FA) per OFDM-frame subject to subcarrier (subc.) selection scheme (equidistant (eq.dst.) or random (rand.)).

\section{B. Influence of number of targets}

Fig. 3 and 4 show the average number of detects and false alarms subject to actual number of targets present. In the simulation for fig. 3 the detector for arbitrarily spaced subcarriers was used whereas in the simulation for fig. 4 the detector for equidistant subcarriers was used.

It can be seen that detection rate decreases with increasing numbers of echos in the received signal. This decrease is less severe for the detector based on equidistant subcarriers than for the detector using arbitrary subcarriers.

The reason for this difference is that both detectors work with a estimation of the power density spectrum and do not exploit phase information. Due to the high sidelobes of the absolute value of the spectral window in the case of nonequidistantly spaced subcarriers it is possible for weak echos to disappear unter the threshold mask. In the case of equidistant subcarriers, that probability is smaller.

\section{Influence of multi user interference}

If multi user interference (MUI) affects only distinct subcarriers, they can be identified and discarded before feeding the data to the detector using a simple threshold discrimination. This holds if the power difference of interfered and interference-free subcarriers is sufficiently large. The results 


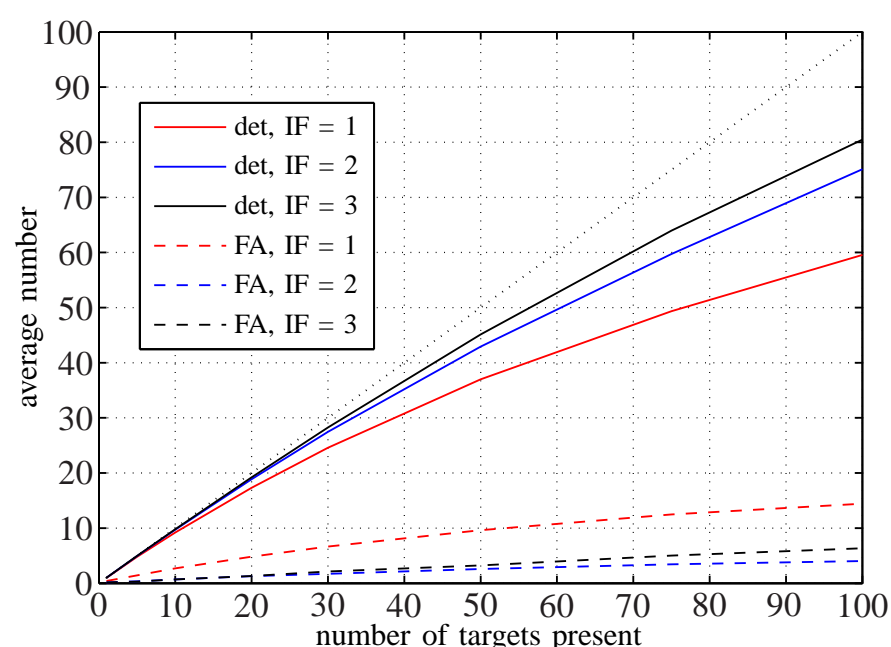

Fig. 3: Detects (det) and false-alarms (FA) per OFDM-frame using arbitrarily spaced subcarriers.

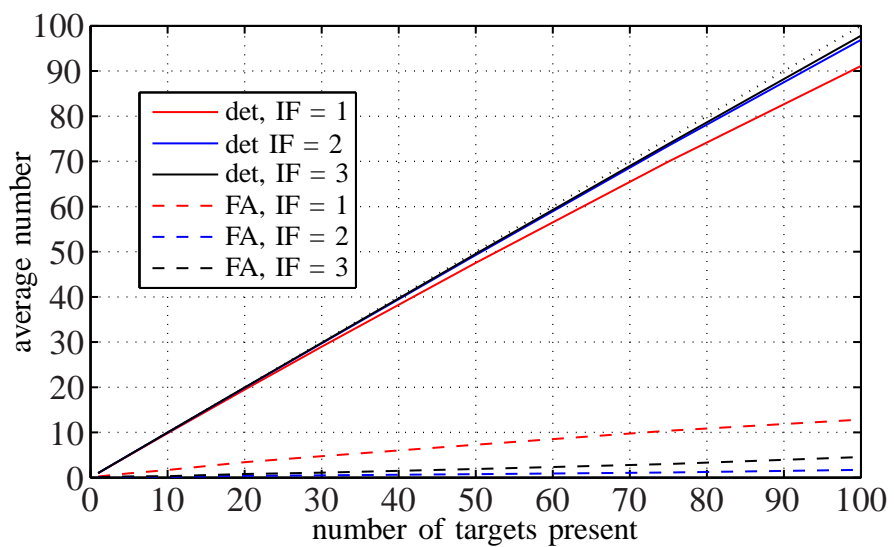

Fig. 4: Detects (det) and false-alarms (FA) per OFDM-frame using equidistant subcarriers.

of a simulation investigating this are shown in fig. 5. They can be explained in the following way: evaluating less subcarriers will at first not change the SNR because signal as well as noise power is discarded. However, the processing gain will be reduced by the ratio of number of discarded to total number of subcarriers. This yields a decreasing detection performance which can be seen in the fig. 5 However, even at very large percentages of discarded subcarriers, still more than half of all targets are detected.

\section{CONCLUSion}

In this paper, a simple multi user access scheme for use in OFDM radar was proposed. The scheme does not mitigate multi user interference (MUI), but instead aims for a low probability of complete signal loss for a user. The advantage of the proposed scheme is that it does neither require any information exchange between users nor does it rely on spectrum sensing techniques. This fact reduces the backbone traffic significantly.

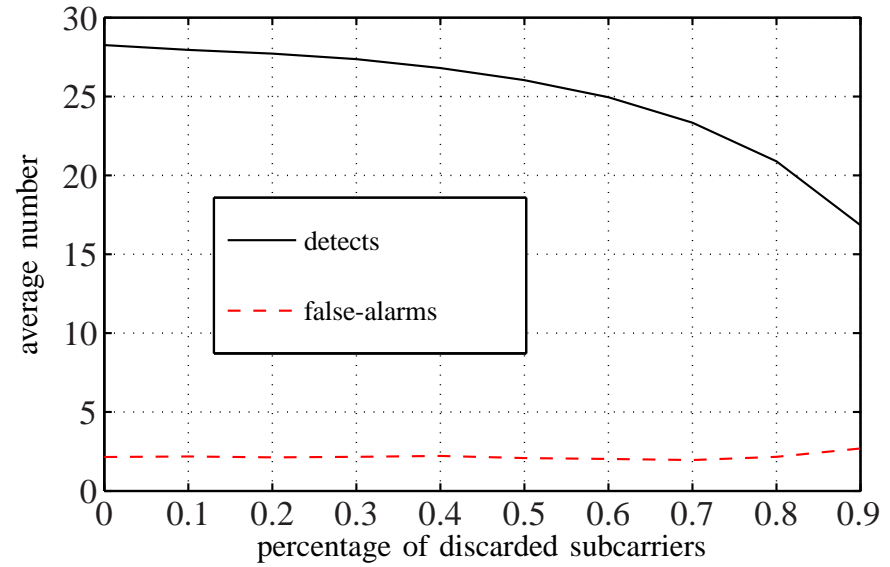

Fig. 5: Detects (det) and false-alarms (FA) subject to number of discarded subcarriers due to interference.

The modified radar detector is able to detect targets on basis of arbitrarily spaced subcarriers. Thus, the work could be extended to non-OFDM schemes e.g. based on filter banks. Furthermore, the proposed radar detector still functions, although at reduced detection rate, even when some of the used subcarriers suffer from MUI. Finally, the detector also is capable of detecting multiple targets.

Compared to a formerly proposed detector based on equidistant subcarriers, there is some performance degradation. However, the formerly proposed system did not provide a multi user access scheme, which is urgently needed in practical scenarios. Furthermore the experienced performance degradation could possibly be compensated on a higher system level, such as target tracking.

The concept proposed can help to further develop OFDM radar an thus to improve road safety.

\section{REFERENCES}

[1] C. Sturm, E. Pancera, T. Zwick, and W. Wiesbeck, "A novel approach to ofdm radar processing," in Radar Conference, 2009 IEEE, may 2009, pp. $1-4$.

[2] M. Braun, C. Sturm, and F. K. Jondral, "Maximum likelihood speed and distance estimation for OFDM radar," in 2010 IEEE Radar Conference. IEEE, 2010, pp. 256-261.

[3] M. Braun, C. Sturm, A. Niethammer, and F. K. Jondral, "Parametrization of joint OFDM-based radar and communication systems for vehicular applications," in 2009 IEEE 20th International Symposium on Personal, Indoor and Mobile Radio Communications, sept. 2009, pp. 3020-3024.

[4] M. Engels, Wireless OFDM Systems. Boston: Kluwer, 2002.

[5] C. Sturm, T. Zwick, and W. Wiesbeck, "An OFDM system concept for joint radar and communications operations," in Vehicular Technology Conference, 2009. VTC Spring 2009. IEEE 69th, april 2009, pp. 1-5.

[6] C. Sturm, T. Zwick, W. Wiesbeck, and M. Braun, "Performance verification of symbol-based ofdm radar processing," in Radar Conference, 2010 IEEE, may 2010, pp. 60-63.

[7] F. Harris, "On the use of windows for harmonic analysis with the discrete fourier transform," Proceedings of the IEEE, vol. 66, no. 1, pp. 51 - 83, jan. 1978.

[8] F. A. M. Frescura, C. A. Engelbrecht, and B. S. Frank, "Significance of periodogram peaks and a pulsation mode analysis of the beta cephei star v403 car," Monthly Notices of the Royal Astronomical Society, vol. 388 , no. 4, pp. 1693-1707, 2008. 